Furthermore, both, axSpA and PsA have been associated with an increased risk of depression.

Objectives: The aim of this project was to assess quality of life, functional impairment in everyday life as well as the prevalence of depression in axSpA and PsA and determine associated factors.

Methods: A questionnaire-based screening tool (FFbH, WHOQOL-BREF, Phq9, GHQ-12) was used to assess 150 patients with PsA and 150 patients with axSpA as well as 300 controls (150 with skin psoriasis only and 150 mechanical back pain patients) for quality of life, functional impairment as well as signs of depression.

Results: Both, SpA and PsA patients had a significantly reduced healthrelated quality of life compared to published normal values as well as psoriasis only patients $(p=0.0003)$. Pain and functional impairment in everyday life correlated highly with perceived overall quality of life $(p<0.01)$. Both, physical health as well as psychological health in turn highly correlated with the presence of depressive symptoms. In total, $35.4 \%$ of SpA and $41.2 \%$ of PsA patients had mild signs of depression, whereas $25 \%$ respectively $28.4 \%$ of SpA and PsA patients had moderate to severe signs of depression. Patients with active disease (BASDAl>4) at the time of evaluation showed significantly more frequent depressive symptoms compared to patients with no or low disease activity $(p<0.0001)$. Female patients had a significantly reduced physical and psychological health-related quality of life compared to male patients $(p=0.0003$, respectively 0.0009$)$. Female SpA and PsA patients also had significantly more depressive symptoms $(p=0.0190)$. In our cohort, also age correlated with perceived quality of life. SpA and PsA patients <45 years of age reported a significantly higher perceived overall quality of life compared to patients $>45$ years $(p<0.0001)$. Quality of life regarding physical health, mental health as well as social relationships was significantly better in younger patients $(p=0.0014, p=0.0109, p=0.0196$ respectively). However, this did not translate into a reduced rate of depressive symptoms in young patients. Body mass index (BMI) was associated with reduced health-related quality of life and psychological wellbeing, but did not correlate with depression. Smoking was neither associated with quality of life nor depression.

Conclusion: Despite treatment many $\mathrm{SpA}$ and PsA patients suffer from a reduced quality of life. Depression constitutes an important comorbidity, which needs to be addressed. Adequate therapy is not only important to reduce irreversible joint damage and disability but may also have a role in avoiding comorbidities such as depression.

Disclosure of Interests: None declared

DOI: 10.1136/annrheumdis-2019-eular.6021

\section{THU0380 LUMBOPELVIC RHYTHM IN PATIENTSWITH AXIAL SPONDYLOARTHRITIS COMPARED WITH LOW BACK PAIN AND HEALTHY SUBJECTS}

Juan L. Garrido-Castro ${ }^{1}$, Inmaculada Concepcion Aranda-Valera' ${ }^{1}$, Sandra AlcarazClariana ${ }^{2}$,Lourdes García-Luque ${ }^{2}$, Daiana Rodriguez-de-Souza²,

Cristina Gonzalez-Navas ${ }^{1}$, Francisco Alburquerque-Sendin ${ }^{2}$, Philip Gardiner ${ }^{3}$, Eduardo Collantes Estevez ${ }^{1} .{ }^{1} I M I B I C$, Cordoba, Spain; ${ }^{2}$ University of Cordoba, Cordoba, Spain; ${ }^{3}$ WHSCT, Londonderry, United Kingdom

Background: Lumbar back pain reduces spinal mobility in patients. Although there are some symptoms that characterise 'inflammatory back pain', these are non-specific and the diagnosis is often delayed. It is not clear if there are kinematic differences between axial Spondyloarthritis (axSpA) and other types of back pain. Inertial motion sensors are now available that can accurately and reliably measure spinal mobility. The ViMove system uses two inertial sensors located at L1 and S1 allowing spinal flexion to be separated into its lumbar and pelvic components. The relation between different parts of the spine during a full flexion movement is known as lumbopelvic rhythm. The trunk sensor measures the total flexion, whilst the contribution of hip is measured by the pelvis sensor leaving lumbar flexion as the difference between the two angles.

Objectives: To analyse relation of lumbopelvic angles in axSpA patients, LBP patients and healthy controls.

Methods: 92 subjects were included in our study: 56 axSpA (from the COSPAR cohort of the Reina Sofia University Hospital), 14 LBP and 22 healthy controls. Inertial based system, the ViMove, was used to measure mobility. axSpA patients were stratified in three groups according their overall mobility using the BASMI: axSpA Low $(<2)$, Med (2-4) and High $(>4)$

Results: Table shows the results of spinal mobility tests in each group. The trunk, pelvis and lumbar angles are expressed in degrees (Mean and SD). cPelvis is the contribution of pelvis to the overall movement as percentage. Three of the five measurements in BASMI are outside the lower back so unlikely to be affected in LBP patients.

\begin{tabular}{lcccccc}
\hline & $\mathrm{n}$ & Trunk & Pelvis & Lumbar & cPelvis & BASMI \\
\hline Control & 22 & 114.8 & 56.7 & $58.0(9.6)$ & $48.7 \%(9.5 \%)$ & 1.7 \\
& & $(13.6)$ & $(15.1)$ & & & $(0.6)$ \\
axSpA & 56 & 103.7 & 56.0 & 47.7 & $54.8 \%$ & 3.1 \\
& & $(19.1)$ & $(14.6)$ & $(17.5)$ & $(15.0 \%)$ & $(1.8)$ \\
LBP & \multirow{2}{*}{14} & 101.3 & 52.8 & 48.5 & $52.0 \%$ & 1.8 \\
& & $(29.5)$ & $(20.0)$ & $(16.4)$ & $(11.4 \%)$ & $(1.0)$ \\
axSpA Low & 21 & 111.3 & 54.2 & $57.0(9.2)$ & $48.5 \%(6.6 \%)$ & 1.5 \\
& & $(13.3)$ & $(10.5)$ & & & $0.5)$ \\
axSpA Med & 22 & 105.9 & 56.2 & 49.6 & $52.6 \%$ & 2.9 \\
& & $(18.8)$ & $(17.1)$ & $(13.1)$ & $(12.3 \%)$ & $(0.5)$ \\
axSpA & \multirow{2}{*}{13} & $87.8(19.4)$ & 58.3 & 29.4 & $68.5 \%$ & 6.0 \\
High & & & $(16.4)$ & $(21.3)$ & $(20.4 \%)$ & $(1.2)$ \\
\hline
\end{tabular}

There were significantly differences in the trunk, lumbar and pelvis angles between control and axSpA groups $(p<0.01)$. The only significant difference between LBP and axSpA was in the lumbar angles $(p<0.05)$.

The left part of the figure shows a reconstruction of the mean flexion angles for each group: these are also represented in the bar graph on the right. The trunk angle is higher in the healthy control group, is similar to the less affected axSpA patients (No significant differences between two groups). The most severely affected axSpA patients show reduced lumbar movement, and the pelvic contribution of the pelvis is also higher than the other two axSpA groups $(p<0.001)$ controls $(p<0.001)$ and LBP $(p<0.01)$.
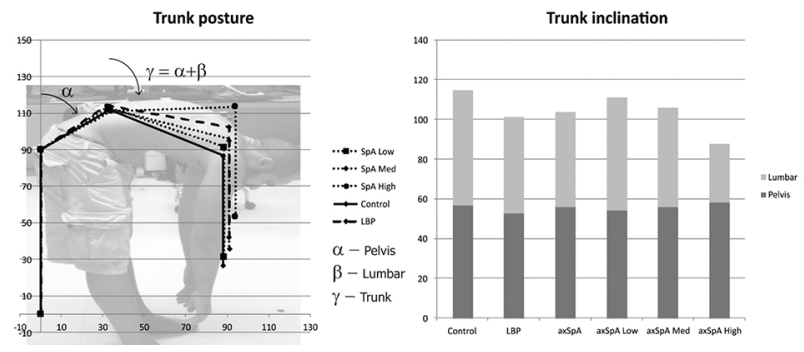

Abstract THU0380 - Figure 1

Conclusion: A reduction in spinal flexion was found in all patient groups, but there are interesting differences in the pattern of movement. LBP patients were characterised by a greater reduction in pelvis movement compared to the axSpA patients, although the final trunk flexion angle is actually lower in axSpA. When we compared more and less affected axSpA patients (according to BASMI), the most severely affected had severe restriction in lumbar movement whilst pelvic movement was increased. Lumbo-pelvic rhythm can also be analysed throughout early, middle and the end of the movement. Investigating spinal kinematics could help us to understand better axSpA and how it affects spinal mobility.

Acknowledgement: This study was funded by FOREUM (www.foreum. org), Junta de Andalucia (PIN-0079-2016) and XXI Research Promotion Program of the University of Córdoba (Spain).

Disclosure of Interests: None declared

DOI: 10.1136/annrheumdis-2019-eular.1053 